\title{
Proctitis following stereotactic body radiation therapy for prostate cancer
}

Daniel Y Joh ${ }^{1 \dagger}$, Leonard N Chen ${ }^{1 \dagger}$, Gerald Porter ${ }^{1}$, Aditi Bhagat ${ }^{1}$, Sumit Sood ${ }^{1}$, Joy S Kim ${ }^{1}$, Rudy Moures ${ }^{1}$, Thomas Yung ${ }^{1}$, Siyuan Lei ${ }^{1}$, Brian T Collins ${ }^{1}$, Andrew W Ju', Simeng Suy ${ }^{1}$, John Carroll ${ }^{3}$, John H Lynch², Anatoly Dritschilo ${ }^{1}$ and Sean P Collins ${ }^{1 *}$

\begin{abstract}
Background: Proctitis after radiation therapy for prostate cancer remains an ongoing clinical challenge and critical quality of life issue. SBRT could minimize rectal toxicity by reducing the volume of rectum receiving high radiation doses and offers the potential radiobiologic benefits of hypofractionation. This study sought to evaluate the incidence and severity of proctitis following SBRT for prostate cancer.

Methods: Between February 2008 and July 2011, 269 men with clinically localized prostate cancer were treated definitively with SBRT monotherapy at Georgetown University Hospital. All patients were treated to 35-36.25Gy in 5 fractions delivered with the CyberKnife Radiosurgical System (Accuray). Rectal bleeding was recorded and scored using the CTCAE v.4. Telangiectasias were graded using the Vienna Rectoscopy Score (VRS). Proctitis was assessed via the Bowel domain of the Expanded Prostate Index Composite (EPIC)-26 at baseline and at 1, 3, 6, 9, 12, 18 and 24 months post-SBRT.
\end{abstract}

Results: The median age was 69 years with a median prostate volume of $39 \mathrm{cc}$. The median follow-up was 3.9 years with a minimum follow-up of two years. The 2-year actuarial incidence of late rectal bleeding $\geq$ grade 2 was $1.5 \%$. Endoscopy revealed VRS Grade 2 rectal telangiectasias in 11\% of patients. All proctitis symptoms increased at one month post-SBRT but returned to near-baseline with longer follow-up. The most bothersome symptoms were bowel urgency and frequency. At one month post-SBRT, $11.2 \%$ and $8.5 \%$ of patients reported a moderate to big problem with bowel urgency and frequency, respectively. The EPIC bowel summary scores declined transiently at 1 month and experienced a second, more protracted decline between 6 months and 18 months before returning to near-baseline at two years post-SBRT. Prior to treatment, $4.1 \%$ of men felt their bowel function was a moderate to big problem which increased to $11.5 \%$ one month post-SBRT but returned to near-baseline at two years post-SBRT.

Conclusions: In this single institution cohort, the rate and severity of proctitis observed following SBRT is low. QOL decreased on follow-up; however, our results compare favorably to those reported for patients treated with alternative radiation modalities. Future prospective randomized studies are needed to confirm these observations.

Keywords: Prostate cancer, SBRT, Rectal endoscopy, Telangiectasias, CyberKnife, Expanded prostate index composite, Bother, Proctitis, Rectal bleeding, Vienna rectoscopy score

\footnotetext{
* Correspondence: SPC9@georgetown.edu

${ }^{\dagger}$ Equal contributors

${ }^{1}$ Department of Radiation Medicine, Georgetown University Medical Center,

3800 Reservoir Road, N.W, Washington, DC 20007, USA

Full list of author information is available at the end of the article
}

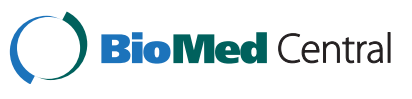

(c) 2014 Joh et al.; licensee BioMed Central Ltd. This is an Open Access article distributed under the terms of the Creative Commons Attribution License (http://creativecommons.org/licenses/by/4.0), which permits unrestricted use, distribution, and reproduction in any medium, provided the original work is properly credited. The Creative Commons Public Domain Dedication waiver (http://creativecommons.org/publicdomain/zero/1.0/) applies to the data made available in this article, unless otherwise stated. 


\section{Background}

Post-treatment bowel function is a primary determinant of quality of life following radiation therapy for prostate cancer [1-5]. Late proctitis occurs at a frequency of 5$20 \%$ with conventionally fractionated treatment [6]. The incidence of proctitis is dependent on the assessment method [7] and whether it is patient or physician reported [8]. Patients with radiation-induced proctitis report increased bowel frequency/urgency, incontinence, bleeding and pain [6]. These symptoms occur months to years after treatment with the large majority of patients reporting symptoms within two years following radiation therapy [9]. Patient characteristics such as age [10], comorbidities [10], hemorrhoids, inflammatory bowel disease [11] and/or anticoagulation [12] may increase an individual's risk of radiation-induced proctitis. Unfortunately, treatment options for radiation proctitis are limited and of unclear clinical benefit [13].

Proctitis is the principle dose-limiting toxicity of prostate radiotherapy [14-16]. Acute proctitis has been attributed to radiation-induced injury to the epithelial lining of the rectal mucosa, leading to mucosal sloughing, acute inflammatory infiltrates, and vascular permeability leading to edema. These early changes are associated with bowel frequency, bowel urgency and rectal pain. Chronic proctitis results from epithelial atrophy and obliterative endarteritis, resulting in tissue ischemia, rectal fibrosis, stricture formation, and neovascularization; this presents clinically with bowel frequency, urgency and rectal bleeding. Historically, endoscopic findings in patients with proctitis include telangiectasia, congested mucosa, and ulcers. Telangiectasias are observed in $20-80 \%$ of patients receiving conventionally fractionated radiation therapy [17].

The risk of proctitis appears to be dependent upon both the total radiation dose and the volume of the rectum in the high dose area [18]. Technical factors such as treatment of the proximal seminal vesicles [19] and expansion of planning target volume (PTV) to compensate for intra-fraction prostate motion [20] may contribute to the severity of rectal toxicities. To minimize bowel toxicity, it is currently recommended that the volume of the rectum receiving $>75$ Gray (Gy) be limited to $<10 \%$ with conventionally fractionated radiation therapy [21,22]. Studies have shown that advanced radiation technologies such as intensity modulated radiation therapy (IMRT) may decrease the dose to the rectum and potentially decrease proctitis [23,24].

Recent data suggest that large radiation fraction sizes are radiobiologically favorable over lower fraction sizes in prostate cancer [25]. The $\alpha / \beta$ for prostate cancer may be as low as $1.5 \mathrm{~Gy}$ [26]. If the $\alpha / \beta$ for prostate cancer is less than 3-5 Gy, which is generally the value accepted for late rectal complications, the linear-quadratic model predicts that delivering large radiation fraction sizes will result in improved local control with a similar rate of bowel complications. Therefore, using large fraction sizes in SBRT provides an attractive modality to leverage the potential radiobiologic benefits of hypofractionation with the minimal invasiveness of an external-beam treatment modality [27]. Furthermore, by reducing the number of treatment visits, hypofractionated therapy can also be logistically favorable for patients and reduce financial burden, ultimately decreasing barriers to care.

The safety and efficacy of SBRT in the treatment of clinically localized prostate cancer has been established in a number of studies [28-33]. The use of large fraction sizes in SBRT offers the potential radiobiological benefits of hypofractionation and potentially may minimize radiation proctitis by reducing the volume of the rectum receiving high radiation doses [34]. The CyberKnife robotic radiosurgical system uses image guidance to track implanted fiducials to account for intrafraction prostatic motion $[35,36]$. This reduces the uncertainty of the location of the prostate and allows treatment to be delivered with a smaller PTV expansion, which may reduce the doses delivered to the anterior rectal wall. The goal of this study is to report the bowel outcomes following SBRT for clinically localized prostate cancer.

\section{Methods}

\section{Patient selection}

Patients eligible for study inclusion had histologicallyconfirmed prostate cancer treated per our institutional protocol. Clinical stage was defined according to the $6^{\text {th }}$ edition of the American Joint Committee on Cancer criteria. Risk groups were defined using the D'Amico criteria [37]. Internal Review Board (IRB)-approval was obtained for retrospective review of data that was prospectively collected in our institutional database.

\section{SBRT treatment planning and delivery}

SBRT was delivered using the CyberKnife robotic radiosurgical system (Accuray Inc., Sunnyvale, CA). The fiducial placement and computed tomography (CT)/magnetic resonance $(\mathrm{MR})$ simulation procedures have been previously described in Lei et al. [36]. The clinical target volume (CTV) was defined as the prostatic capsule and proximal seminal vesicles (to the point where the seminal vesicles separate). To cover areas of potential ECE, the expansion from the CTV to the PTV was $5 \mathrm{~mm}$ in all directions except $3 \mathrm{~mm}$ posteriorly into the rectum. There was no difference in the target volume delineation for different risk groups. Fiducial-based tracking was used to account for interfraction and intrafraction prostate motion [35]. Treatment planning was performed using Multiplan (Accuray Inc., Sunnyvale, CA). Patients were treated with 35 or 36.25 Gy of radiotherapy delivered in 5 fractions of 
7-7.25 Gy each to the PTV, administered over a timespan of 1-2 weeks. This corresponds to a tumor equivalent dose in 2-Gy fractions (EQD2) of approximately 8590 Gy assuming an $\alpha / \beta$ ratio of 1.5 . The rectum was contoured as a solid structure from the anus (at the level of the ischial tuberosities) to the rectosigmoid flexure and evaluated with dose-volume histogram analysis during treatment planning using Multiplan (Accuray Inc., Sunnyvale, CA) inverse treatment planning. Rectal volume receiving 36 Gy was limited to $\leq 1 \mathrm{cc}$. Assuming an $\alpha / \beta$ of 3 Gy for late bowel complications, this is biologically equivalent to approximately 74 Gy administered in 2 Gy fractions. The rectal dose-volume histogram (DVH) goals were $<50 \%$ rectal volume receiving $50 \%$ of the prescribed dose, $<20 \%$ receiving $80 \%$ of the dose, $<10 \%$ receiving $90 \%$ of the dose, and $<5 \%$ receiving $100 \%$ of the dose. Typical dose distributions have been previously described $[28,34,38]$. Patients were placed on a low-residual diet and given enemas prior to simulation and treatment delivery to maximize the potential distance between the prostate and the rectal wall and minimize intrafraction prostate motion.

\section{Follow-up and statistical analysis}

Rectal bleeding was prospectively documented at followup visits using the National Cancer Institute (NCI) Common Toxicity Criteria (CTC) version 4.0. Acute rectal bleeding was defined as experiencing toxicity within 6 months of radiation therapy. Late rectal bleeding was defined as occurring at least 6 months after delivery of radiation therapy. Grade 1 represents minimal bleeding not requiring medications for symptom control. Grade 2 indicates rectal bleeding requiring new medication (i.e. steroid suppository) or minor laser coagulation. Telangiectasia were graded using the Vienna Rectoscopy Score (VRS): Grade 1 (a single telangiectasia), Grade 2 (multiple non-confluent telangiectasia) and Grade 3 (multiple confluent telangiectasia) [39].

Proctitis was assessed via the bowel domain of the Expanded Prostate Index Composite (EPIC)-26 at baseline and at $1,3,6,9,12,18$ and 24 months [40]. The timing of the quality of life (QOL) assessments was relative to the last day of SBRT treatment. The EPIC-26 bowel domain includes five questions related to individual symptoms (questions 6a-e: urgency, frequency, pain, bloody stool, incontinence) and one question (question 7) related to overall bother (degree of interference or annoyance caused by bowel symptoms [41].

Wilcoxon signed-rank test and chi-square analysis were used to assess differences in ongoing quality of life scores in comparison to baseline. For each EPIC question, the responses were grouped into three clinically relevant categories (no problem, very small-small problem and moderate to big problem). To statistically compare changes between time points, the levels of responses were assigned a score and the significance of the mean changes in the scores was assessed by Wilcoxon rank test. EPIC scores for the bowel domain and its individual questions range from $0-100$ with lower values representing worsening bowel symptoms. The minimally important difference (MID) in EPIC score was defined as a change of one-half standard deviation (SD) from the baseline [42]. To limit the effect of attrition bias, statistical analysis was limited to time points in which $\geq 80 \%$ of the patient data were available.

\section{Results}

From February 2008 to July 2011, 269 prostate cancer patients were treated per our institutional SBRT monotherapy protocol (Table 1). The median follow-up was 3.9 years. The median age was 69 years and $44.2 \%$ were of non-Caucasian ancestry. Comorbidities were common with $35 \%$ were taking anticoagulants (including aspirin) prior to treatment. The median prostate volume was 39 cc. By D’Amico classification, 99 patients were low-, 143 intermediate-, and 27 high-risk. 16.4\% also received androgen deprivation therapy (ADT) at the discretion of the treating Urologist. 83.3\% of the patients were treated with 36.25 Gy in five 7.25 Gy fractions.

A total of $61(22.7 \%)$ patients reported rectal bleeding after SBRT, with $28(10.4 \%)$ patients reporting acute bleeding and 38 patients (14.1\%) reporting late bleeding. Overall, Grade 2 acute and late rectal bleeding were observed in $0(0 \%)$ and $4(1.5 \%)$ of patients, respectively. There was no Grade 3 or higher rectal bleeds. The majority of acute rectal bleeding occurred within 1-month post-SBRT treatment (79\%). The bleeding completely resolved in the majority of the patients by the subsequent follow-up visit. Likewise, the majority of late rectal bleeding was observed at one specific follow-up appointment and did not persist on subsequent follow-ups.

Seventy-three patients had one or more rectal endoscopies during the follow-up period. Endoscopy was performed as a part of normal colorectal cancer screening or if patient had clinically significant rectal bleeding. The median interval from completion of SBRT to endoscopy was 24 months (3 months to 53 months). On endoscopy, rectal telangiectasias were found in 8 (11.0\%) patients. All 8 patients were observed to have multiple non-confluent telangiectasias (VRS Grade 2) [38]. No patient had confluent or circumferential telangiectasia. Minor laser coagulation was performed in three patients. No rectal ulcerations, strictures, or fistulas were observed.

At baseline, 1.9\% of our cohort reported some level of annoyance due to bloody stools, however no patient felt it was a moderate to big problem (Table 2). The mean changes in EPIC bloody stool bother scores from baseline to 2 years of follow-up are shown in Table 3 . The 
Table 1 Baseline patient characteristics and treatment

\% Patients $(\mathrm{N}=\mathbf{2 6 9})$

\begin{tabular}{lll}
\hline Age $(\mathbf{y} / \mathbf{0})$ & Median 69 (44-90) & \\
& $<60$ & $8.20 \%$ \\
$60-69$ & $42.40 \%$ \\
& $70-79$ & $41.30 \%$ \\
& $\geq 80$ & $8.20 \%$
\end{tabular}

Race

$\begin{array}{ll} & \text { White } \\ & \text { Black } \\ & \text { Other } \\ \text { Prostate volume (cc) } & \text { Median 39.04 } \\ & (11.56-138.69)\end{array}$

Charlson comorbidity index (CCl)

$\begin{array}{ll}0 & 66.9 \% \\ 1 & 22.7 \% \\ 2 & 6.7 \% \\ \geq 3 & 3.7 \%\end{array}$

Body mass index

\begin{tabular}{lll} 
& $\mathrm{BMI}<25$ & $21.9 \%$ \\
& $25 \leq \mathrm{BMI}<30$ & $47.2 \%$ \\
& $30 \leq \mathrm{BMI}<35$ & $21.9 \%$ \\
& $\mathrm{BMI} \geq 35$ & $8.9 \%$ \\
Use of anticoagulants & Yes & $35.3 \%$ \\
Risk group & $\mathrm{No}$ & $64.7 \%$ \\
& & \\
& Low & $36.80 \%$ \\
Hormonal therapy & Intermediate & $53.20 \%$ \\
& High & $10.00 \%$ \\
& & \\
Dose & Yes & $16.40 \%$ \\
& No & $83.60 \%$ \\
& & \\
& 36.25 Gy & $16.40 \%$ \\
& 35 Gy & $0.40 \%$ \\
\hline
\end{tabular}

mean EPIC bloody stool bother score was 99.5 at baseline (Table 3). Bloody stool bother increased following treatment with the mean score decreasing to 96.6 at 1 month post-treatment (mean change, -2.9) $(p=0.0002)$ (Table 3, Figure $1 \mathrm{~d}$ ). However, only $1.2 \%$ of patients felt that that bloody stools were a moderate to big problem at 1 month following treatment (Table 2). Although bloody stool bother declined quickly, a second late increase in bloody stool bother was observed with the mean bloody stool bother score decreasing to 97.4 at 18 months (mean change from baseline, -2.1) $(\mathrm{p}=0.0066)$ (Table 3, Figure 1d). Both declines met the threshold for clinically significant change $(\mathrm{MID}=1.7)$. However, only $1.2 \%$ of patients felt that that bloody stools were a moderate to big problem at 12 months following treatment (Table 2). By two years following SBRT, bloody stool bother returned to near-baseline with bloody stool bother score of 98.1 (mean change from baseline, -1.4). By two years post-SBRT, no patient felt that blood stools was a moderate to big problem (Table 2).

Baseline proctitis symptoms were uncommon in our patients (Table 2). All proctitis symptoms increased at one month post-SBRT. The most bothersome symptoms were bowel urgency and frequency. At one month post SBRT, $11.2 \%$ and $8.5 \%$ of patients reported moderate to big problems with bowel urgency and frequency, respectively (Table 2). 9.7\% of patients required anti-diarrheals within the first month postSBRT.

Likewise, the EPIC bowel summary score declined transiently at 1 month (mean change, -9.8) (Table 3). In addition, a second more protracted decline occurred between 6 and 18 months (mean change from baseline at 12 months, -3.5). Bowel declines at 1 month and 12 months were statistically significant $(p<0.0001)$; however, only the 1 month change met the threshold for clinically significant change $(\mathrm{MID}=4.6)$ (Figure 2$)$. The EPIC bowel summary score returned to near-baseline at two years post-SBRT (mean change from baseline, -1.6). Individual bowel symptoms changed with time in a similar manner (Figure 1).

Overall bowel bother showed a similar pattern as the bowel summary score. At baseline, 24.0\% of patients reported some level of annoyance due to bowel symptoms with $4.1 \%$ of patients feeling that bowel function was a moderate to big problem (Table 4). The mean EPIC bowel bother score was 90.7 at baseline (Table 3). Overall bowel bother worsened posttreatment and the mean score decreased to 75 at 1 month (mean change, -14.8) (Table 3, Figure 3). However, only $11.5 \%$ of patients felt that their bowel function was a moderate to big problem at 1 month following SBRT (Table 4). Bowel bother scores worsened over a second, more protracted time period (Figure 3). Once again, only the first decline met the threshold for clinically significant change (MID $=9.8)$. Only $7 \%$ of patients felt that bowel symptoms were a moderate to big problem at 12 month following treatment (Table 4). By two years following SBRT, overall bowel bother returned to near baseline with a bowel bother score of 89.8 (mean change from baseline, -0.9) (Table 3) and 3.3\% of patients feeling bowel symptoms were a moderate to big problem (Table 4). 
Table 2 Bowel symptoms following SBRT for prostate cancer

\begin{tabular}{|c|c|c|c|c|c|c|c|c|c|}
\hline & & Start & 1 & 3 & 6 & 9 & 12 & 18 & 24 \\
\hline \multicolumn{10}{|l|}{ Bowel urgency } \\
\hline No problem & & $77.2 \%$ & $49.8 \%$ & $62.0 \%$ & $60.2 \%$ & $64.5 \%$ & $62.3 \%$ & $64.7 \%$ & $66.8 \%$ \\
\hline Very small-small problem & & $21.0 \%$ & $39.0 \%$ & $33.7 \%$ & $34.1 \%$ & $29.8 \%$ & $31.6 \%$ & $29.5 \%$ & $31.1 \%$ \\
\hline \multirow[t]{2}{*}{ Moderate-big problem } & & $1.9 \%$ & $11.2 \%$ & $4.3 \%$ & $5.6 \%$ & $5.6 \%$ & $6.1 \%$ & $5.8 \%$ & $2.1 \%$ \\
\hline & $p$ value & & $<0.0001$ & 0.0002 & $<0.0001$ & 0.0002 & 0.0001 & 0.0004 & 0.01568 \\
\hline \multicolumn{10}{|l|}{ Bowel frequency } \\
\hline No problem & & $82.0 \%$ & $57.0 \%$ & $71.9 \%$ & $71.5 \%$ & $70.6 \%$ & $72.5 \%$ & $76.3 \%$ & $77.4 \%$ \\
\hline Very small-small problem & & $15.7 \%$ & $34.5 \%$ & $25.0 \%$ & $25.3 \%$ & $23.4 \%$ & $23.0 \%$ & $18.8 \%$ & $19.7 \%$ \\
\hline \multirow[t]{2}{*}{ Moderate-big problem } & & $2.2 \%$ & $8.5 \%$ & $3.1 \%$ & $3.2 \%$ & $6.0 \%$ & $4.5 \%$ & $4.9 \%$ & $2.9 \%$ \\
\hline & $p$ value & & $<0.0001$ & 0.0079 & 0.0038 & 0.0031 & 0.0214 & 0.0169 & 0.2508 \\
\hline \multicolumn{10}{|l|}{ Incontinence } \\
\hline No problem & & $94.8 \%$ & $79.5 \%$ & $85.2 \%$ & $86.7 \%$ & $88.3 \%$ & $85.2 \%$ & $85.7 \%$ & $89.5 \%$ \\
\hline Very small-small problem & & $4.1 \%$ & $17.0 \%$ & $14.5 \%$ & $11.2 \%$ & $9.7 \%$ & $12.3 \%$ & $11.6 \%$ & $8.8 \%$ \\
\hline \multirow[t]{2}{*}{ Moderate-big problem } & & $1.1 \%$ & $3.5 \%$ & $0.4 \%$ & $2.0 \%$ & $2.0 \%$ & $2.5 \%$ & $2.7 \%$ & $1.7 \%$ \\
\hline & $p$ value & & $<0.0001$ & 0.0033 & 0.0021 & 0.03 & 0.0001 & 0.0001 & 0.0147 \\
\hline \multicolumn{10}{|l|}{ Bloody stools } \\
\hline No problem & & $98.1 \%$ & $90.8 \%$ & $96.9 \%$ & $95.6 \%$ & $96.0 \%$ & $96.3 \%$ & $94.2 \%$ & $94.2 \%$ \\
\hline Very small-small problem & & $1.9 \%$ & $8.1 \%$ & $2.3 \%$ & $3.6 \%$ & $2.4 \%$ & $2.5 \%$ & $5.4 \%$ & $5.8 \%$ \\
\hline \multirow[t]{2}{*}{ Moderate-big problem } & & $0.0 \%$ & $1.2 \%$ & $0.8 \%$ & $0.8 \%$ & $1.6 \%$ & $1.2 \%$ & $0.4 \%$ & $0.0 \%$ \\
\hline & $p$ value & & 0.0002 & 0.1677 & 0.0934 & 0.0574 & 0.0906 & 0.0066 & 0.0258 \\
\hline \multicolumn{10}{|c|}{ Pain (abdominal, pelvic or rectal) } \\
\hline No problem & & $86.1 \%$ & $76.8 \%$ & $89.8 \%$ & $86.7 \%$ & $86.7 \%$ & $88.5 \%$ & $86.6 \%$ & $90.3 \%$ \\
\hline Very small-small problem & & $12.4 \%$ & $17.8 \%$ & $7.8 \%$ & $10.4 \%$ & $9.7 \%$ & $8.2 \%$ & $12.1 \%$ & $7.1 \%$ \\
\hline \multirow[t]{3}{*}{ Moderate-big problem } & & $1.5 \%$ & $5.4 \%$ & $2.3 \%$ & $2.8 \%$ & $3.6 \%$ & $3.3 \%$ & $1.3 \%$ & $2.5 \%$ \\
\hline & $p$ value & & 0.0002 & 0.5516 & 0.6606 & 0.232 & 0.5713 & 0.8565 & 0.7498 \\
\hline & $N=$ & 267 & 259 & 255 & 249 & 248 & 244 & 224 & 238 \\
\hline
\end{tabular}

Patient-reported responses to EPIC-26 questions 6A (Urgency to have a bowel movement), 6B (Frequency of bowel movements), 6C (Losing control of your stools), 6D (Bloody stools) and 6E (Abdominal, pelvic or rectal pain).

\section{Discussion}

Proctitis following prostate cancer radiotherapy is a critical quality of life issue [1-4] and the principle doselimiting toxicity [14-16]. Currently, there is limited data on proctitis following SBRT for prostate cancer. A better understanding of bowel symptoms following SBRT would enable clinicians to provide more realistic expectations to patients as they weigh their treatment options. Our prior paper reported the prevalence, severity and overall incidence of bowel frequency/urgency, rectal pain and rectal bleeding following SBRT [28]. However, it is reliant on physician reporting which may under report the incidence of bowel symptoms [8] and provides no information on the associated bother $[43,44]$. In this study, we utilized the EPIC-26 bowel domain to evaluate bowel symptoms but also assess related bother.

Stereotactic body radiation therapy (SBRT) offers to minimize radiation-associated rectal toxicity by reducing

Table 3 Changes in EPIC bowel summary and overall bowel bother scores following SBRT for prostate cancer

\begin{tabular}{|c|c|c|c|c|c|c|c|c|c|c|c|}
\hline \multirow[t]{2}{*}{ EPIC criteria } & \multirow[t]{2}{*}{ Baseline } & \multicolumn{2}{|l|}{1 Month } & \multicolumn{2}{|l|}{6 Month } & \multicolumn{2}{|c|}{12 Month } & \multicolumn{2}{|c|}{18 Month } & \multicolumn{2}{|c|}{24 Month } \\
\hline & & Change & S.D. & Change & S.D. & Change & S.D. & Change & S.D. & Change & S.D. \\
\hline Bloody stool bother & 99.5 & -2.9 & 12.26 & -1.1 & 8.54 & -1.3 & 9.86 & -2.1 & 10.93 & -1.4 & 8.03 \\
\hline Bowel summary & 94.8 & -9.8 & 17.89 & -3.2 & 12.21 & -3.5 & 13.04 & -3.3 & 13.40 & -1.6 & 10.73 \\
\hline Bowel bother & 90.7 & -14.8 & 26.98 & -2.6 & 20.82 & -5.5 & 23.95 & -3.5 & 20.91 & -0.9 & 18.86 \\
\hline
\end{tabular}




\section{a}

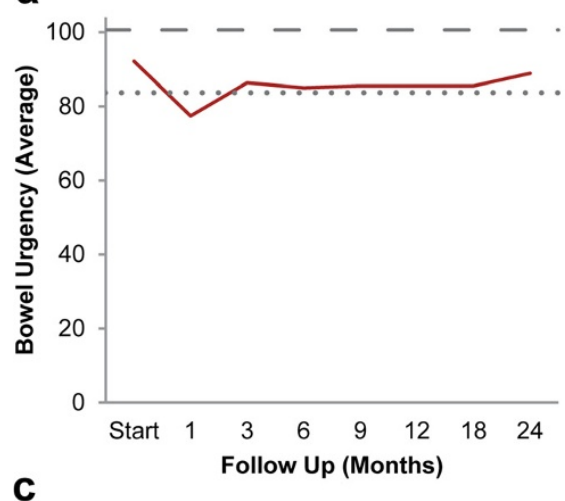

C

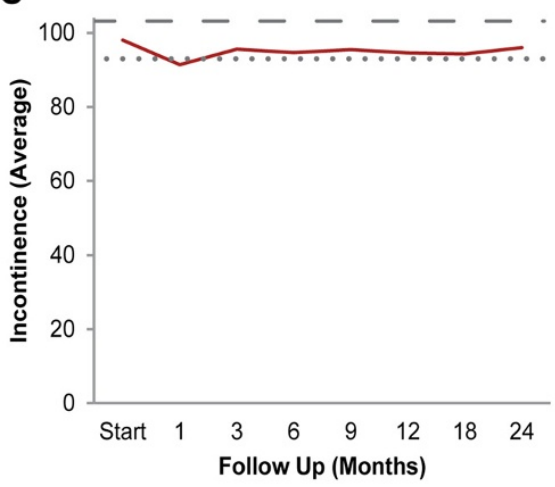

b

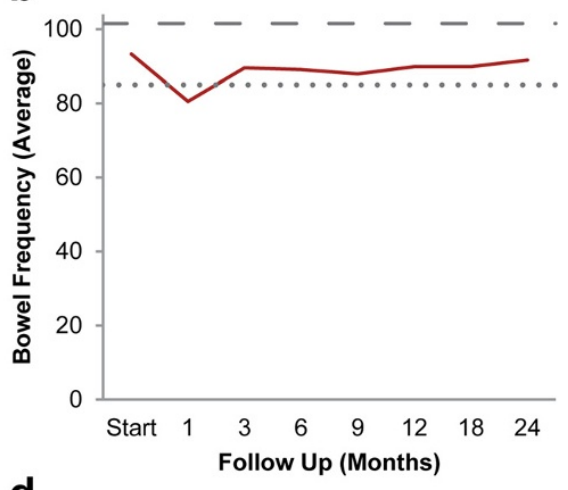

d

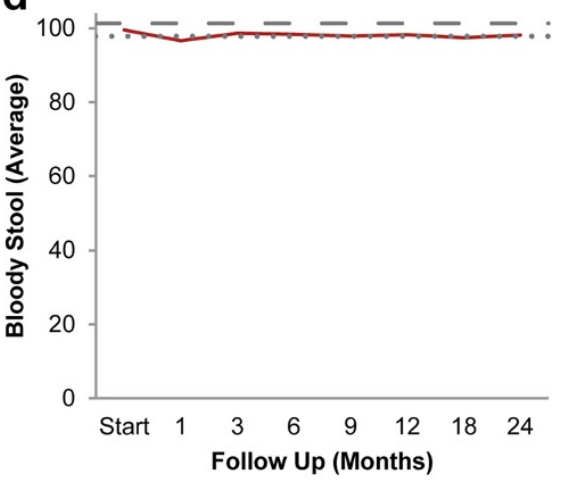

e

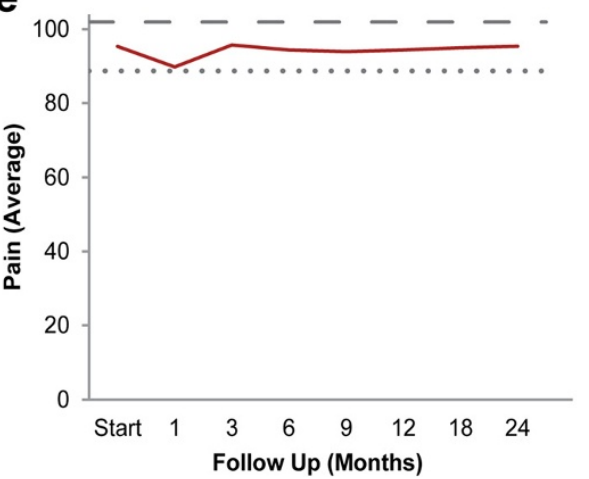

Figure 1 Individual EPIC-26 bowel symptoms (Questions 6a-e). Average individual symptom EPIC bother scores at baseline and following SBRT for prostate cancer: (a) urgency to have a bowel movement-Question 6a of the EPIC-26; (b) increase frequency of bowel movements- Question 6b of the EPIC-26; (c) losing control of stools- Question 6c of the EPIC-26; (d) bloody stools- Question 6d of the EPIC-26; (e) abdominal/pelvic/rectal pain- Question 6e of the EPIC-26. Thresholds for clinically significant changes in scores ( $1 \frac{1}{2}$ standard deviation above and below the baseline) are marked with dashed lines. EPIC scores range from 0-100 with higher values representing a more favorable health-related QOL.

the volume of rectum receiving high radiation doses. The overall incidence of post-SBRT rectal bleeding in this series was $23 \%$. The low rate of late Grade $\geq 2$ rectal bleeding (1.5\%) seen in this study is consistent with the results from a prior review by our institution [28] and results from other institutions [45,32] which all report a rate of late Grade $\geq 2$ rectal bleeding of $<5 \%$. In fact, many of the bleeds were acute and transient suggestive of acute anal irritation or hemorrhoid exacerbation due to bowel frequency [38]. Furthermore, the incidence of telangiectasia in patients who completed post-SBRT rectal endoscopy for the assessment of rectal bleeding or cancer screening was $11 \%$ which is significantly lower than the rates reported in prospective studies that looked at endoscopic outcomes after conventionally fractionated radiation therapy $[17,46,47]$.

These findings are particularly significant given that rectal bleeding is one of the principle dose-limiting 


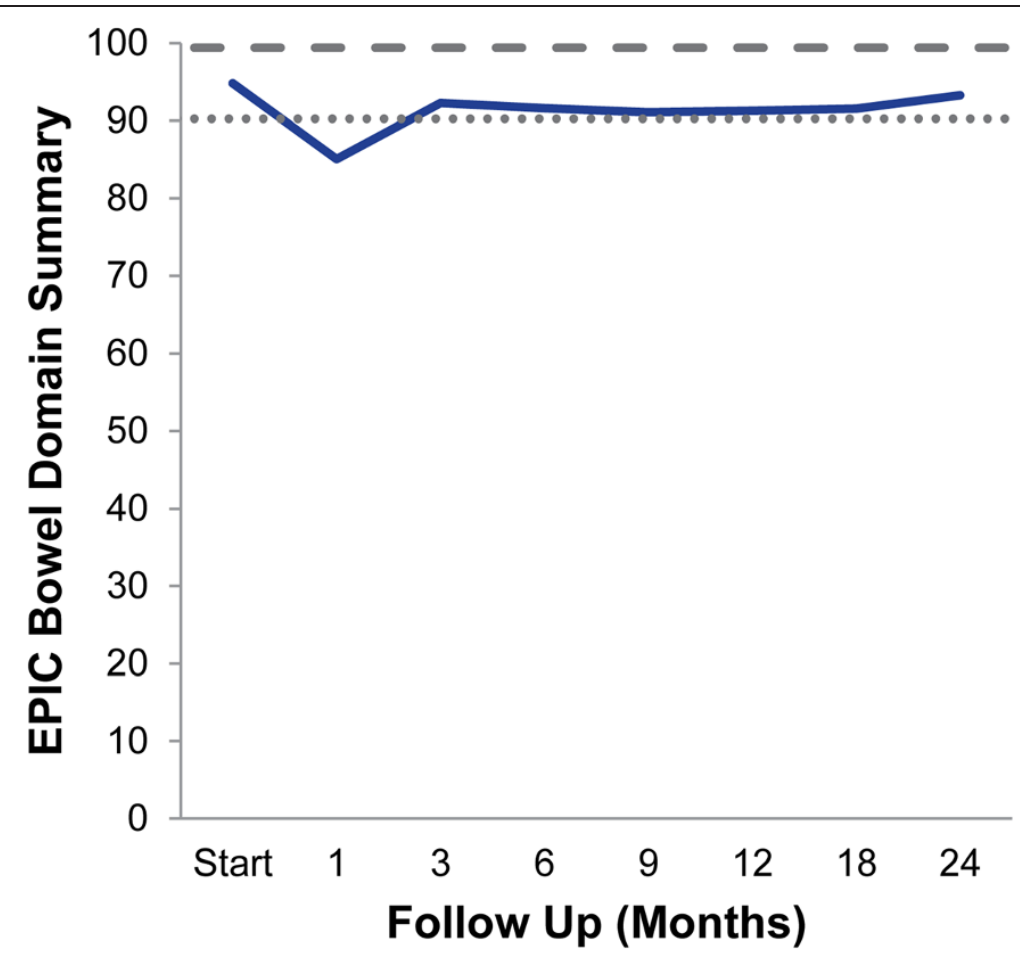

Figure 2 Average EPIC bowel summary scores at baseline and following SBRT for prostate cancer. Thresholds for clinically significant changes in scores ( $1 / 2$ standard deviation above and below the baseline) are marked with dashed lines. EPIC scores range from 0-100 with higher values representing a more favorable health-related QOL.

toxicities, and is thus a potential barrier to administering radiation at appropriately therapeutic levels. In the work by Sanda et al, the proportion of patients reporting rectal bleeding as a moderate-big problem on the EPIC 26 survey progressively increased over time (reaching 5\% at 12 and 24 months), ultimately manifesting as a late complication post-treatment. However, we observed that with SBRT rectal bleeding occurred at lower rates and presented as a relatively earlier complication: the incidence of patients reporting rectal bleeding as a moderatebig problem in this study reached a peak at 9 months at $1.6 \%$, and by 24 months the incidence dropped to null. Additionally, in our series the rate of Grade 2-3 late rectal bleeding was $1.5 \%$. This compares favorably to the $5 \%$ $15 \%$ seen with contemporary high-dose IMRT $[24,48]$. We speculate that this reassuring profile with SBRT may be a result of the enhanced accuracy with fiducial tracking and narrowed target volumes, thus enhancing our efforts to spare normal tissue from inadvertent irradiation.

The pattern seen in bowel QOL after SBRT in our study is similar to the pattern seen after conventionally fractionated radiotherapy, proton therapy or brachytherapy. The bowel QOL score is at its lowest 1 or 2 months after treatment, but improves slowly thereafter to near baseline by 1-2 years after treatment [3-5]. In our series, most moderate to big problems were seen at 1 month post-SBRT with approximately $10 \%$ of patients reporting moderate to big problems with bowel urgency and/or frequency (Figure 4). This compares favorably with 15$19 \%$ incidence of moderate to severe bowel urgency and frequency seen two months after conventionally fractionated external beam radiation therapy or brachytherapy [3].

Table 4 Overall bowel bother following SBRT for prostate cancer (Patient-reported responses to Question 7 of the EPIC-26)

\begin{tabular}{|c|c|c|c|c|c|c|c|c|c|}
\hline & & Start & 1 & 3 & 6 & 9 & 12 & 18 & 24 \\
\hline No problem & & $76.0 \%$ & $44.6 \%$ & $68.0 \%$ & $69.1 \%$ & $63.7 \%$ & $63.5 \%$ & $66.1 \%$ & $70.4 \%$ \\
\hline Very small-small problem & & $19.9 \%$ & $43.8 \%$ & $28.9 \%$ & $26.9 \%$ & $30.2 \%$ & $29.5 \%$ & $29.5 \%$ & $26.3 \%$ \\
\hline \multirow[t]{3}{*}{ Moderate-big problem } & & $4.1 \%$ & $11.5 \%$ & $3.1 \%$ & $4.0 \%$ & $6.0 \%$ & $7.0 \%$ & $4.5 \%$ & $3.3 \%$ \\
\hline & $p$ value & - & $<0.0001$ & 0.1044 & 0.0828 & 0.0051 & 0.0005 & 0.0172 & 0.3499 \\
\hline & $N=$ & 267 & 260 & 256 & 249 & 248 & 244 & 224 & 240 \\
\hline
\end{tabular}




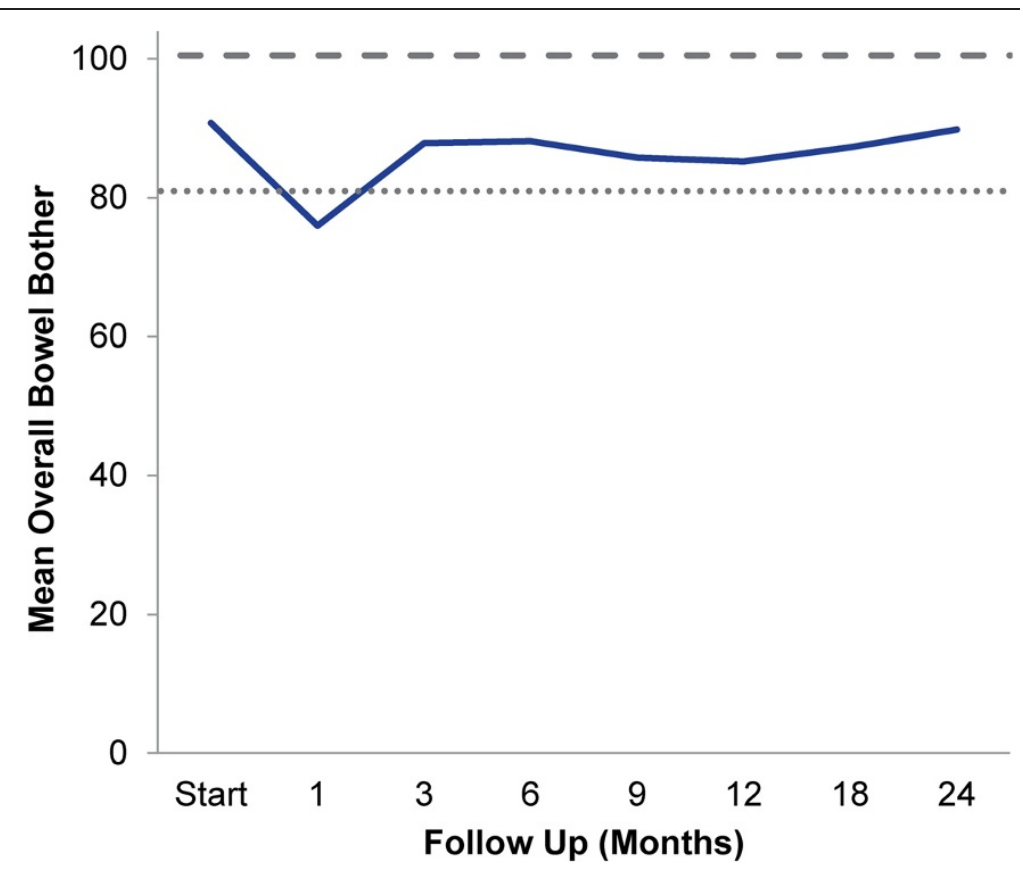

Figure 3 Overall bowel bother score (baseline and following SBRT; Question 7 of EPIC-26). Thresholds for clinically significant changes in scores ( $1 / 2$ standard deviation above and below the baseline) are marked with dashed lines. EPIC scores range from 0-100 with higher values representing a more favorable health-related QOL.

Unlike conventional modalities, this increase in bowel bother was transient and returned to near baseline by 3 months post-SBRT $[3,5]$. A second transient increase in bowel bother occurred at 12 months with approximately $7 \%$ of patients reporting moderate to big problems. Unlike the pattern seen with conventional modalities, post-SBRT bowel bother returned to near baseline by two years $[3,5]$. The mean bowel summary score change from baseline to 24 months in this study was -1.6. This change compares favorably to that seen at 24 months with conventionally fractionated IMRT and proton therapy -7.4 and -3.7 , respectively [3-5,14].

The present study has several identifiable limitations. The patient population was derived from a single highvolume institution cohort that can limit the translation of our work to the general population. Nevertheless, our work utilized a fairly large patient population with excellent follow-up rates, and was heterogeneous with respect to ethnicity and risk stratification, and considered key patient characteristics such as comorbidity, body mass

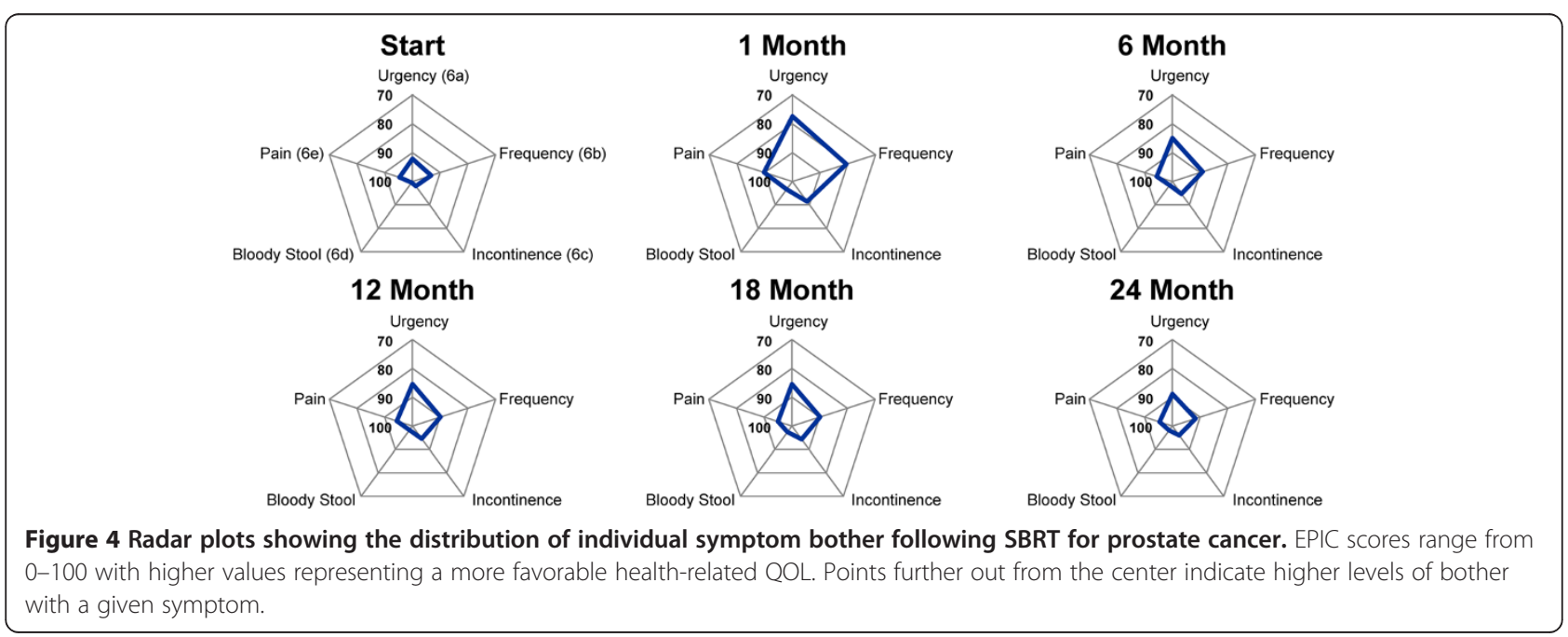


index, and use of anticoagulants and hormonal therapy. It is conceivable, however, that institutions without the same experience may have a learning curve before they can achieve similar results. Also noteworthy is that our analysis was conducted without a concurrent comparator arm and thus must be carefully weighed against previously published work. Reassuringly, given that the observed toxicity rates in the acute phase are within range of previous studies, the superior toxicity profile at two years follow-up with SBRT compared to other modalities offers intriguing insight to guide subsequent trials for a more comprehensive assessments of SBRTrelated proctitis in the future.

\section{Conclusions}

In this single institution cohort, the rate and severity of proctitis observed following SBRT is low. QOL tended to improve with longer follow-up and was near baseline at two years post-SBRT. Our results compare favorably to those reported for patients treated with alternative radiation modalities. Future prospective randomized studies are needed to confirm these observations.

\section{Consent}

This study was a retrospective review of prospectively collected data that was approved by the Georgetown University Institutional Review Board.

\section{Abbreviations \\ ADT: Androgen deprivation therapy; $C$ : Computed tomography; $C T C$ : Common toxicity criteria; CTV: Clinical target volume; DVH: Dose-volume histogram; EQD2: Equivalent dose in 2-Gy fractions; EPIC: Expanded prostate index composite; GTV: Gross target volume; Gy: Gray; IMRT: Intensity modulated radiation therapy; IRB: Internal review board; PTV: Planning target volume; QoL: Quality of life; MID: Minimally important difference; MR: Magnetic resonance; NCl: National cancer institute; SD: Standard deviation; SBRT: Stereotactic body radiation therapy; VRS: Vienna rectoscopy score.}

\section{Competing interests}

SP Collins and BT Collins serve as clinical consultants to Accuray Inc. The Department of Radiation Medicine at Georgetown University Hospital receives a grant from Accuray to support a research coordinator. The other authors declare that they have no competing interests.

\section{Authors' contributions}

DJ and LC are lead authors, who participated in data collection, data analysis, manuscript drafting, table/figure creation, and manuscript revision. GP participated in data analysis and manuscript drafting. AB, SS, RM, and JK aided in the quality of life data collection and maintaining the patient database. TY aided in clinical data collection. SL is the dosimetrist who developed the majority of patients' treatment plans, and contributed to the dosimetric data analysis and interpretation. $\mathrm{BC}$ and $\mathrm{AJ}$ participated in the design and coordination of the study. SS is a senior author who collected the dosimetric data, participated in its analysis, and helped draft the manuscript. JC, $A D$, and $J L$ are senior authors who aided in drafting the manuscript. SC was the principal investigator who initially developed the concept of the study and the design, aided in data collection, drafted and revised the manuscript. All authors read and approved the final manuscript.

\section{Acknowledgements}

The authors gratefully acknowledge generous funding support from the James and Theodore Pedas Family Foundation and NIH Grant P30CA051008.

\section{Author details}

'Department of Radiation Medicine, Georgetown University Medical Center, 3800 Reservoir Road, N.W, Washington, DC 20007, USA. ² Department of Urology, Georgetown University Hospital, Washington, DC 20007, USA. ${ }^{3}$ Department of Gastroenterology, Georgetown University Hospital, Washington, DC 20007, USA. ${ }^{4}$ Department of Radiation Oncology, East Carolina University, Greenville, NC 27834, USA.

Received: 24 July 2014 Accepted: 26 November 2014

Published online: 12 December 2014

\section{References}

1. Bacon CG, Giovannucci E, Testa M, Glass TA, Kawachi I: The association of treatment-related symptoms with quality-of-life outcomes for localized prostate carcinoma patients. Cancer 2002, 94:862-871.

2. Geinitz H, Zimmermann FB, Thamm R, Erber C, Muller T, Keller M, Busch R, Molls M: Late rectal symptoms and quality of life after conformal radiation therapy for prostate cancer. Radiother Oncol 2006, 79:341-347.

3. Sanda MG, Dunn RL, Michalski J, Sandler HM, Northouse L, Hembroff L, Lin X, Greenfield TK, Litwin MS, Saigal CS, Mahadevan A, Klein E, Kibel A, Pisters LL, Kuban D, Kaplan I, Wood D, Ciezki J, Shah N, Wei JT: Quality of life and satisfaction with outcome among prostate-cancer survivors. N Engl J Med 2008, 358:1250-1261

4. Gray PJ, Paly JJ, Yeap BY, Sanda MG, Sandler HM, Michalski JM, Talcott JA, Coen JJ, Hamstra DA, Shipley WU, Hahn SM, Zietman AL, Bekelman JE, Efstathiou JA: Patient-reported outcomes after 3-dimensional conformal, intensity-modulated, or proton beam radiotherapy for localized prostate cancer. Cancer 2013, 119:1729-1735.

5. Hoppe BS, Michalski JM, Mendenhall NP, Morris CG, Henderson RH, Nichols RC, Mendenhall WM, Williams CR, Regan MM, Chipman JJ, Crociani CM, Sandler HM, Sanda MG, Hamstra DA: Comparative effectiveness study of patient-reported outcomes after proton therapy or intensity-modulated radiotherapy for prostate cancer. Cancer 2013, 120:1076-1082.

6. Garg AK, Mai WY, McGary JE, Grant WH 3rd, Butler EB, Teh BS: Radiation proctopathy in the treatment of prostate cancer. Int I Radiat Oncol Biol Phys 2006, 66:1294-1305

7. Lund JA, Kaasa S, Wibe A, Widmark A, Fransson P. Late radiation effects to the rectum and anus after treatment for prostate cancer; validity of the LENT/SOMA score. Acta Oncol 2013, 52:727-735.

8. Sonn GA, Sadetsky N, Presti JC, Litwin MS: Differing perceptions of quality of life in patients with prostate cancer and their doctors. J Urol 2009, 182:2296-2302.

9. Eifel PJ, Levenback C, Wharton JT, Oswald MJ: Time course and incidence of late complications in patients treated with radiation therapy for FIGO stage IB carcinoma of the uterine cervix. Int J Radiat Oncol Biol Phys 1995, 32:1289-1300

10. Hamstra DA, Stenmark MH, Ritter T, Litzenberg D, Jackson W, Johnson S, Albrecht-Unger L, Donaghy A, Phelps L, Blas K, Halverson S, Marsh R, Olson $K$, Feng FY: Age and comorbid illness are associated with late rectal toxicity following dose-escalated radiation therapy for prostate cancer. Int J Radiat Oncol Biol Phys 2013, 85:1246-1253.

11. Willett CG, Ooi CJ, Zietman AL, Menon V, Goldberg S, Sands BE, Podolsky DK Acute and late toxicity of patients with inflammatory bowel disease undergoing irradiation for abdominal and pelvic neoplasms. Int I Radiat Oncol Biol Phys 2000, 46:995-998.

12. Choe KS, Jani AB, Liauw SL: External beam radiotherapy for prostate cancer patients on anticoagulation therapy: how significant is the bleeding toxicity? Int I Radiat Oncol Biol Phys 2010, 76:755-760.

13. Leiper K, Morris Al: Treatment of radiation proctitis. Clin Oncol (R Coll Radiol) 2007, 19:724-729.

14. Kuban DA, Tucker SL, Dong L, Starkschall G, Huang EH, Cheung MR, Lee AK, Pollack A: Long-term results of the M. D. Anderson randomized doseescalation trial for prostate cancer. Int J Radiat Oncol Biol Phys 2008, 70:67-74.

15. Dearnaley DP, Sydes MR, Graham JD, Aird EG, Bottomley D, Cowan RA, Huddart RA, Jose CC, Matthews JH, Millar J, Moore AR, Morgan RC, Russell JM, Scrase CD, Stephens RJ, Syndikus I, Parmar MK: Escalateddose versus standard-dose conformal radiotherapy in prostate cancer: first results from the MRC RT01 randomised controlled trial. Lancet Oncol 2007. 8:475-487. 
16. Al-Mamgani A, van Putten WL, Heemsbergen WD, van Leenders GJ, Slot A Dielwart MF, Incrocci L, Lebesque JV: Update of Dutch multicenter doseescalation trial of radiotherapy for localized prostate cancer. Int J Radiat Oncol Biol Phys 2008, 72:980-988.

17. Goldner G, Tomicek B, Becker G, Geinitz H, Wachter S, Zimmermann F, Wachter-Gerstner N, Reibenwein J, Glocker S, Bamberg M, Feldmann H, Pötzi R, Molls M, Pötter R: Proctitis after external-beam radiotherapy for prostate cancer classified by Vienna Rectoscopy Score and correlated with EORTC/RTOG score for late rectal toxicity: results of a prospective multicenter study of 166 patients. Int J Radiat Oncol Biol Phys 2007, 67:78-83.

18. Michalski JM, Gay H, Jackson A, Tucker SL, Deasy JO: Radiation dosevolume effects in radiation-induced rectal injury. Int J Radiat Oncol Biol Phys 2010, 76:S123-S129

19. Bayman NA, Wylie JP: When should the seminal vesicles be included in the target volume in prostate radiotherapy? Clin Oncol (R Coll Radiol) 2007, 19:302-307.

20. Dawson LA, Mah K, Franssen E, Morton G: Target position variability throughout prostate radiotherapy. Int J Radiat Oncol Biol Phys 1998, 42:1155-1161

21. Tucker SL, Dong L, Michalski JM, Bosch WR, Winter K, Cox JD, Purdy JA Mohan R: Do intermediate radiation doses contribute to late rectal toxicity? An analysis of data from radiation therapy oncology group protocol 94-06. Int J Radiat Oncol Biol Phys 2012, 84:390-395.

22. Hamstra DA, Conlon AS, Daignault S, Dunn RL, Sandler HM, Hembroff AL, Zietman AL, Kaplan I, Ciezki J, Kuban DA, Wei JT, Sanda MG, Michalski JM: Multi-institutional prospective evaluation of bowel quality of life after prostate external beam radiation therapy identifies patient and treatment factors associated with patient-reported outcomes: the PROSTQA experience. Int J Radiat Oncol Biol Phys 2013, 86:546-553

23. Zelefsky MJ, Levin EJ, Hunt M, Yamada Y, Shippy AM, Jackson A, Amols HI: Incidence of late rectal and urinary toxicities after three-dimensional conformal radiotherapy and intensity-modulated radiotherapy for localized prostate cancer. Int J Radiat Oncol Biol Phys 2008, 70:1124-1129.

24. Michalski JM, Yan Y, Watkins-Bruner D, Bosch WR, Winter K, Galvin JM, Bahary JP, Morton GC, Parliament MB, Sandler HM: Preliminary toxicity analysis of 3-dimensional conformal radiation therapy versus intensity modulated radiation therapy on the high-dose arm of the Radiation Therapy Oncology Group 0126 prostate cancer trial. Int J Radiat Oncol Biol Phys 2013, 87:932-938.

25. Fowler JF: The radiobiology of prostate cancer including new aspects of fractionated radiotherapy. Acta Oncol 2005, 44:265-276.

26. Brenner DJ, Hall EJ: Fractionation and protraction for radiotherapy of prostate carcinoma. Int J Radiat Oncol Biol Phys 1999, 43:1095-1101.

27. Ritter M, Forman J, Kupelian P, Lawton C, Petereit D: Hypofractionation for prostate cancer. Cancer J 2009, 15:1-6.

28. Chen LN, Suy S, Uhm S, Oermann EK, Ju AW, Chen V, Hanscom HN, Laing S, Kim JS, Lei S, Batipps GP, Kowalczyk K, Bandi G, Pahira J, McGeagh KG, Collins BT, Krishnan P, Dawson NA, Taylor KL, Dritschilo A, Lynch JH, Collins SP: Stereotactic body radiation therapy (SBRT) for clinically localized prostate cancer: the Georgetown University experience. Radiat Oncol 2013, 8:58.

29. Katz AJ, Santoro M, Diblasio F, Ashley R: Stereotactic body radiotherapy for localized prostate cancer: disease control and quality of life at 6 years. Radiat Oncol 2013, 8:118.

30. King CR, Collins SP, Fuller D, Wang P, Kupelian P, Steinberg M, Katz A: Health Related Quality of Life after Stereotactic Body Radiotherapy for Localized Prostate Cancer: Results from a Multi-Institutional Consortium of Prospective Trials. Int J Radiat Oncol Biol Phys 2013, 87:939-945.

31. King CR, Freeman D, Kaplan I, Fuller D, Bolzicco G, Collins SP, Meier R, Wang J, Kupelian P, Steinberg M, Katz A: Stereotactic Body Radiotherapy for Localized Prostate Cancer: Pooled Analysis of Multi-Insitutional Prospective Trials. Radiat Ther Oncol 2013, 109:217-221.

32. King CR, Brooks JD, Gill H, Presti JC Jr: Long-term outcomes from a prospective trial of stereotactic body radiotherapy for low-risk prostate cancer. Int J Radiat Oncol Biol Phys 2011, 82:877-882.

33. McBride SM, Wong DS, Dombrowski JJ, Harkins B, Tapella P, Hanscom HN, Collins SP, Kaplan ID: Hypofractionated stereotactic body radiotherapy in low-risk prostate adenocarcinoma: Preliminary results of a multiinstitutional phase 1 feasibility trial. Cancer 2012, 118:3681-3690.
34. Ju AW, Suy S, Lei S, Wang H, Oermann EK, Sherer BA, Uhm S, Hanscom HN Kim JS, Lynch JH, Dritschilo A, Collins SP: Hypofractionated stereotactic body radiation therapy as monotherapy for intermediate-risk prostate cancer. Radiation Oncology. Radiat Oncol 2013, 8:30.

35. Xie $Y$, Djajaputra D, King CR, Hossain S, Ma L, Xing L: Intrafractional motion of the prostate during hypofractionated radiotherapy. Int J Radiat Oncol Biol Phys 2008, 72:236-246.

36. Lei S, Piel N, Oermann EK, Chen V, Ju AW, Dahal KN, Hanscom HN, Kim JS, Yu X, Zhang G, Collins BT, Jha R, Dritschilo A, Suy S, Collins SP: SixDimensional Correction of Intra-Fractional Prostate Motion with CyberKnife Stereotactic Body Radiation Therapy. Front Oncol 2011, $1: 48$.

37. D'Amico AV, Whittington R, Malkowicz SB, Schultz D, Blank K, Broderick GA, Tomaszewski JE, Renshaw AA, Kaplan I, Beard CJ, Wein A: Biochemical outcome after radical prostatectomy, external beam radiation therapy, or interstitial radiation therapy for clinically localized prostate cancer. Jama 1998, 280:969-974.

38. Sood S, Ju AW, Wang H, Lei S, Uhm S, Zhang G, Suy S, Carroll J, Lynch J, Dritschilo A, Collins SP: Rectal endoscopy findings following stereotactic body radiation therapy for clinically localized prostate cancer. Radiat Oncol 2013, 8:197.

39. Wachter S, Gerstner N, Goldner G, Potzi R, Wambersie A, Potter R: Endoscopic scoring of late rectal mucosal damage after conformal radiotherapy for prostatic carcinoma. Radiother Oncol 2000, 54:11-19.

40. Wei JT, Dunn RL, Litwin MS, Sandler HM, Sanda MG: Development and validation of the expanded prostate cancer index composite (EPIC) for comprehensive assessment of health-related quality of life in men with prostate cancer. Urology 2000, 56:899-905.

41. Reeve BB, Potosky AL, Willis GB: Should function and bother be measured and reported separately for prostate cancer quality-of-life domains? Urology 2006, 68:599-603.

42. Norman GR, Sloan JA, Wyrwich KW: Interpretation of changes in healthrelated quality of life: the remarkable universality of half a standard deviation. Med Care 2003, 41:582-592.

43. Gore JL, Gollapudi K, Bergman J, Kwan L, Krupski TL, Litwin MS: Correlates of bother following treatment for clinically localized prostate cancer. J Urol 2010, 184:1309-1315.

44. Litwin MS, Gore JL, Kwan L, Brandeis JM, Lee SP, Withers HR, Reiter RE: Quality of life after surgery, external beam irradiation, or brachytherapy for early-stage prostate cancer. Cancer 2007, 109:2239-2247.

45. Katz AJ, Santoro M, Ashley R, Diblasio F, Witten M: Stereotactic body radiotherapy for organ-confined prostate cancer. BMC Urol 2010, 10:1.

46. Ippolito E, Massaccesi M, Digesu C, Deodato F, Macchia G, Pirozzi GA, Cilla S, Cuscuna D, Di Lallo A, Mattiucci GC, Mantini G, Pacelli F, Valentini V, Cellini N, Ingrosso M, Morganti AG: Early proctoscopy is a surrogate endpoint of late rectal toxicity in prostate cancer treated with radiotherapy. Int J Radiat Oncol Biol Phys 2012, 83:e191-e195.

47. van Lin EN, Kristinsson J, Philippens ME, de Jong DJ, van der Vight LP, Kaanders JH, Leer JW, Visser AG: Reduced late rectal mucosal changes after prostate three-dimensional conformal radiotherapy with endorectal balloon as observed in repeated endoscopy. Int J Radiat Oncol Biol Phys 2007, 67:799-811.

48. Spratt DE, Pei X, Yamada J, Kollmeier MA, Cox B, Zelefsky MJ: Long-term survival and toxicity in patients treated with high-dose intensity modulated radiation therapy for localized prostate cancer. Int $J$ Radiat Oncol Biol Phys 2013, 85:686-692.

\section{doi:10.1186/s13014-014-0277-4}

Cite this article as: Joh et al:: Proctitis following stereotactic body radiation therapy for prostate cancer. Radiation Oncology 2014 9:277. 